DE92 004017

\title{
THE INFLUENCE OF PRESSURE ON SOLUTE RETENTION IN LIQUID CHROMATOGRAPHY
}

Victoria L. McGuffin" and Christine E. Evans ${ }^{1}$

Department of Chemistry, Michigan State University, East Lansing, MI 48824, USA

\section{DISCLAIMER}

\section{KEY WORDS}

capillary liquid chromatography

on-column detection

laser fluorescence detection

solute retention

pressure effects

\begin{abstract}
This report was prepared as an account of work sponsored by an agency of the United States Government. Neither the United States Government nor any agency thereof, nor any of their employees, makes any warranty, express or implied, or assumes any legal liability or responsibility for the accuracy, completeness, or usefulness of any information, apparatus, product, or process disclosed, or represents that its use would not infringe privately owned rights. Reference herein to any specific commercial product, process, or servic by trade name, trademark, manufacturer, or otherwise does not necessarily constitute or imply its endorsement, recommendation, or favoring by the United States Government or any agency thereof. The views and opinions of authors expressed herein do not necessarily state or reflect those of the United States Government or any agency thereof.
\end{abstract}

\section{SUMMARY}

In this investigation, multiple on-column detectors are utilized to measure the retention of model solutes directly on a packed, capillary column. The absolute pressure on the column is controlled using a restrictor at the column exit, while the pressure gradient and volumetric flowrate are maintained constant. Measurements obtained under reversedphase conditions indicate that the local capacity factor changes considerably with local pressure under typical operating conditions. These results are somewhat surprising since the mobile-phase solvents used for liquid chromatography are generally considered to be incompressible.

\section{INTRODUCTION}

Pressure has long been recognized to have a significant influence on the movement of solute zones in chromatographic separations (1-3). As early as 1964, Giddings (1) suggested that increased separating potential could be realized by compressing the gaseous mobile phase to form so-called dense gases. Due to the high compressibility and the significant pressure range possible, the gaseous mobile phase may exhibit solvent properties that are pressure dependent (4-6). These variations in solvating power with pressure reach an extreme when the mobile phase is a supercritical fluid, where compressibility is very high and small changes in pressure can lead to large variations in fluid density. In the case of liquid chromatography, the compressibility is quite small and the influence of pressure on solute retention is often assumed to be negligible. In contrast to separatione utilizing gas or supercritical fluids, however, common operating pressures for liquid chromatographic separations are considerably

1 Present address: School of Chemical Sciences, University of Illinois, Urbana, IL 61801, USA

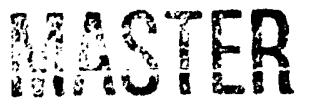


greater, typically 100-350 bar. These inlet pressures, combined with small but finite compressibilities, lead to changes in the mobile-phase density of $1-5 \%$ under typical chromatographic conditions. These variations in density, and the concomitant change in the mobile-phase solvating power, may result in solute retention that is pressure dependent.

Recently, Martire has developed a unified theoretical approach to understanding this phenomenon in all mobile-phase fluids (7). Unfortunately, only a limited number of systematic experimental investigations have been reported in the literature for liquid chromatographic separations. Under extreme conditions (1380 bar), Rogers et al. (8-9) measure as much as a three-fold increase in capacity factor and a significant change in selectivity for separations utilizing an adsorption/ion-exchange mechanism. Likewise, Tanaka et al. (10) observe a $12 \%$ variation in capacity factor for reversed-phase separations under ionization control for much more modest pressures (207 bar). Normial-phase measurements by Katz et al. (11) indicate a decrease in retention, which the authors attribute to an increase in temperature in the column interior. Thus, contrary to common misconceptions, these few experimental results indicate that variations in the absolute pressure may have a significant effect on solute retention.

These findings have interesting implications for the variation in solute retention under commor operating conditions. Because flow is a pressure-driven process in chromatographic separations, a pressure gradient is present on the column at all times. Thus, the capacity factor measured at the column outlet is most likely an avsiage of the retention behavior along the coiumn (7). The determination of fundamental retention parameters may be, therefore, more complicated than previously recognized.

The on-column detection scheme developed in our laboratory (12) may be used to advantage in evaluating this phenomenon. The measurement of local solute retention directly on the chromatographic colurnn allows the systematic evaluation of the influence of local pressure. By varying the inlet pressure while maintaining constant pressure gradient conditions, the local retention may be directly correlated with the local pressure on the column. Moreover, the use of small diameter, packed capillary columns minimizes variations in temperature within the column that may arise from viscous flow. A homologous series of coumarin-derivatized fatty acics have been chosen as the model solutes for this study. When separated using a pure methanoi mobile phase, the alkyl portion of the molecuie appears to partition into the stationary phase, while the coumarin moiety resides in the mobile phase (13). Thus, these solutes are not only chromatographically and spectroscopically well-behaved, but they probe the most universal type of molecular interactions, induced-dipole induced-dipole (14). Utilizing 
this experimental design, systematic measurements of the dependence of local pressure on solute retention should find general applicability for liquid chromatographic separations.

\section{EXPERIMENTAL METHODS}

\subsection{Reagents}

Saturated fatty acid standards ranging from $n-C_{10: 0}$ to $n-C_{20: 0}$ (Sigma Chemical Co.) are derivatized with 4-bromomethyl-?-methoxycoumarin as described previously (15). An anhydrous mixture (1:1) of sodium sulfate and potassium bicarbonate $(0.005 \mathrm{~g})$ is combined with dibenzo-18-crown-6 $(0.0036 \mathrm{~g})$ in a slurry formed using $0.2 \mathrm{~mL} \mathrm{dry}$ acetone. After stirring, a $0.500 \mathrm{~mL}$ aliquot of $10^{-3} \mathrm{M}$ stock solution containing the fatty acid standards is added together with the coumarin reagent $(0.0027 \mathrm{~g})$. The reaction is allowed to proceed in the dark at $50^{\circ} \mathrm{C}$ for 2.5 hours with intermittent stirring. Individual fatty acid derivatives are then isolated and purified using a conventional-scale octadecylsilica column (ODS-224, Applied Biosystems, Inc.) using methanol as the mobile phase. The resulting fractions are evaporated in a stream of nitrogen at $40^{\circ} \mathrm{C}$ and redissolved in methanol at a final concentration of $5 \times 10^{-4} \mathrm{M}$. All organic solvents used in this study are high-purity, distilled-in-glass grade (Burdick \& Jackson Division, Baxter Healthcare Corp.).

\subsection{Chromatographic System}

The experimental system utilized for this study is shown schematically in Figure 1. The mobile phase is delivered by a single-piston reciprocating pump (Model 114M, Beckman Instruments), operated in the constant-pressure mode. The sample is introduced by means of a $1.0-\mu \mathrm{L}$ injection valve (Valco Instruments Co.), and the effluent is subsequently split between the microcolumn and a capillary $(1: 90)$, resulting in a nominal flowrate of $0.70 \mu \mathrm{L} / \mathrm{min}$ and an injection volume of $11 \mathrm{~nL}$. A 25.7-cm length of opentubular capillary $(50 \mu \mathrm{m}$ i.d.) is utilized to connect the injector to the microcolumn. This arrangement makes possible the placement of detectors in the high-pressure regioir near the column inlet.

The microcolumn is fabricated from $0.200 \mathrm{~mm}$ i.d. fused-silica capillary tubing (HewlettPackard), terminated with a quartz wool frit at a length of approximately $43.9 \mathrm{~cm}$. Prior to packing, the polyimide coating is removed from the capillary at $5 \mathrm{~cm}$ intervals to facilitate on-column detection. A slurry of 3- $\mu$ r. spherical octadecylsilica packing material (MicroPak SP, Varian Associates) is then introduced under moderate pressure. The resulting microcolumn has a plate height $(H)$ of $9.5 \mu \mathrm{m}$, a total porosity $\left(\varepsilon_{T}\right)$ of 0.58 , and a flow resistance parameter ( $\left.\phi^{\prime}\right)$ of 550 under standard test conditions (16). A 20 $\mu \mathrm{m}$ i.d. fused-silica capillary (Polymicro Technologies) is attached at the column outlet. 
During the course of this study, the lengths of the restricting and splitting capillaries are altered, so that the volumetric flowrate and split ratio remain constant while the inlet pressure is varied from approximately 100 to 350 bar.

\subsection{Detection System}

This detection system utilizes laser-induced fluorescence to prube solute zones at six positions directly on the liquid chromatographic microcolumn (12-14). A continuouswave He-Cd laser (Model 3112-10S, Omnichrome), with $10 \mathrm{~mW}$ power at $325 \mathrm{~nm}$, is utilized as the excitation source for each fluorescence detector. Laser radiation is transmitted to the microcolumn via small-diameter, UV-grade optical fibers (100 $\mu \mathrm{m}$, Polymicro Technologies), and fluorescence emission is collected in a right-angle geometry using optical fibers of larger diameter $(500 \mu \mathrm{m}$, Polymicro Technologies). As illustrated in Figure 1, the fluorescent emission is transmitted from alternate detectpr blocks to one of two identical detection systems. The combined emission is filtered to remove scattered and second-order $r_{i}$ diation, and is focused onto the entrance slit of a monochromator (Model H1061, Instruments SA). The fluorescence emission from the derivatized fatty acids at $420 \mathrm{~nm}$ is detected by a photomultiplier tube (R1463, Hamamatsu Corp.), and the resulting photocurrent is amplified (100 nAV, $0.06 \mathrm{~s}$ time constant) and converted to the digital domain (Model 3405/5716, Data Translation). All data acquisition is performed at a rate of $5 \mathrm{~Hz}$ using the Forth-based programming language Asyst (Macmillan Software, Keithley Instruments) with an IBM PC-XT computer.

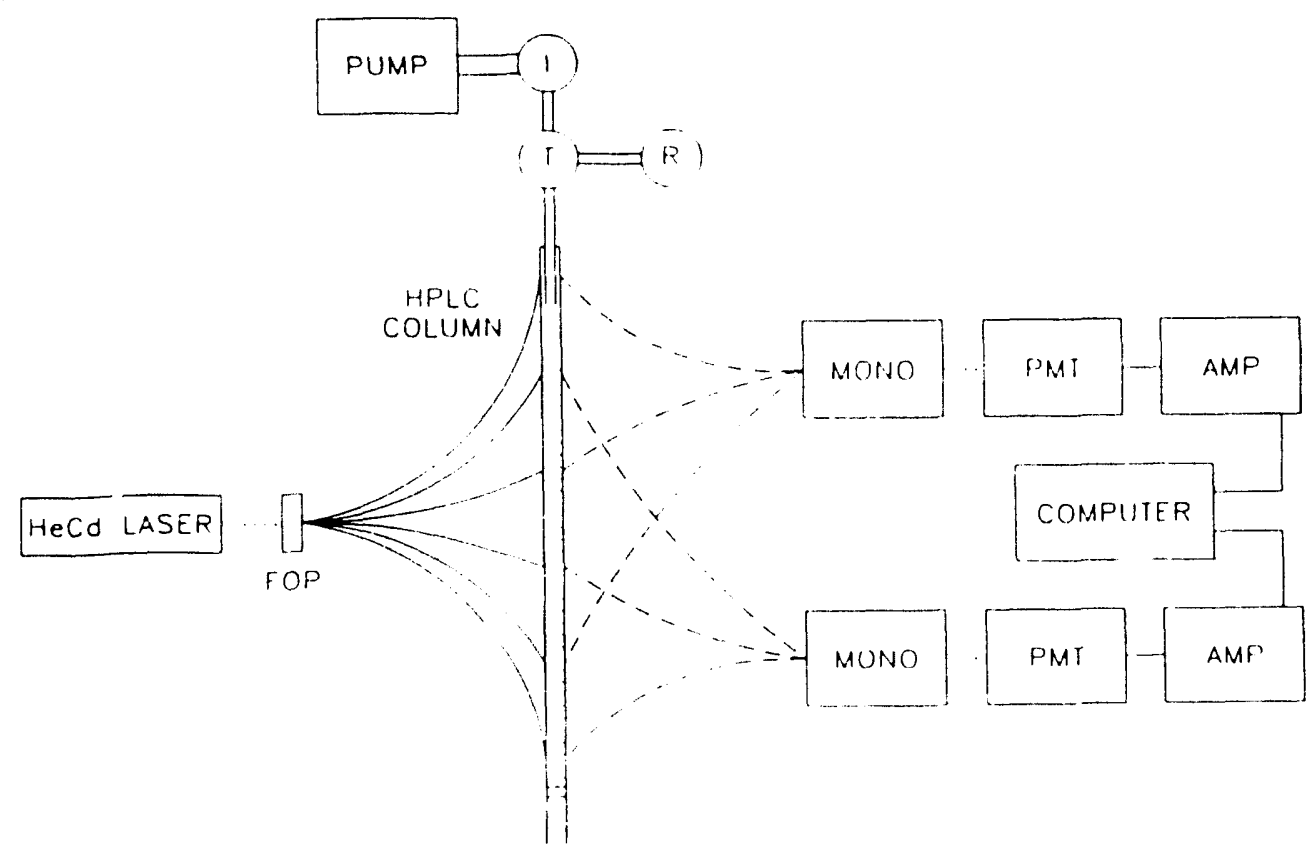

Figure 1

Schematic diagram of detection systern allowing simultaneous measurement at six points along the chromatographic column with only two monochromator/photomultiplier systems. I: injection valve; $\mathrm{T}$ : splitting tee; $\mathrm{R}$ : restricting capillary; MONO: monochromator; PMT: photomultiplier; AMP: current-to-voltage converter/amplifier. 


\subsection{Calculations}

Statistical moments are chosen as the most accurate means to determine the retention time of each solute zone (17). Although rigorously defined as integrals, statistical moments have been calculated based on finite summation of the fluorescence intensity as a function of time $[l(t)]$.

$M_{0}=\Sigma l(t) d t$

$M_{1}=\sum t l(t) d t / M_{0}$

Physically, the zeroth moment $\left(M_{0}\right)$ represents the area, while the first moment $\left(M_{1}\right)$ is the centroid of the solute zone. All calculations use a minimum of 50 data points uniformly distributed across the zone profile.

For single-mode detection, the solute retention time is equal to the first statistical moment calculated at each detector. Thus, the capacity factor may be evaluated directly from the first moment of the solute zone and that of a nonretained zone, corresponding with the elution of acetone. In dual-mode detection, the retention time in the region between detectors is calculated as the difference in the first statistical moments $\left(M_{1}\right)$ evaluated at each detector. Thus, the solute capacity factor may be evaluated either as the average behavior between the injector and the point of detection using a single detector (single mode), or isolated to a specific region of the column between the two detectors (dual mode).

\section{RESULTS AND DISCUSSION}

Experimental evaluation of the pressure dependence of solute capacity factor is accomplished by systematically increasing the inlet pressure from 102 to 337 bar, while maintaining a constant pressure differential of 102 bar along the column. By measuring the capacity factor using single- and dual-mode detection, the average retention between the injector and the point of detection as well as the local retention between detectors may be determined directly on the column. In addition, measurements are performed simultaneously at all detector positions in the high-pressure region of the column, allowing the evaluation of the local capacity factor at several points for a single injection. Experimental measurements performed in this manner allow the direct, in situ determination of solute retention as a function of the local pressure.

Initial evaluation of the pressure dependence is accomplished by measuring the solute capacity factor as a function of distance from the column inlet. Since the locai pressure is expected to decrease linearly with distance, in situ measurements at a given inlet pressure allow a preliminary indication of any variation in retention with the average pressure encountered by the solute. As seen in Figure 2, single-mode measurements 
(Figure 2, top) show a clear increase in capacity factor with distance along the column. In contrast, dual-mode determinations (Figure 2, bottom) are relatively constant with distance. Thus, preliminary results are conflicting; single-mode measurements indicating an apparent decrease in capacity factor with local pressure and dual-mode measurements showing no variation in retention with local pressure. In addition, previous measurements on is similar column suggested that solute capacity factor actually increases with local pressure (14). These results are, therefore, inconclusive and indicate that additional factors may be affecting the retention of solutes with distance along the column.

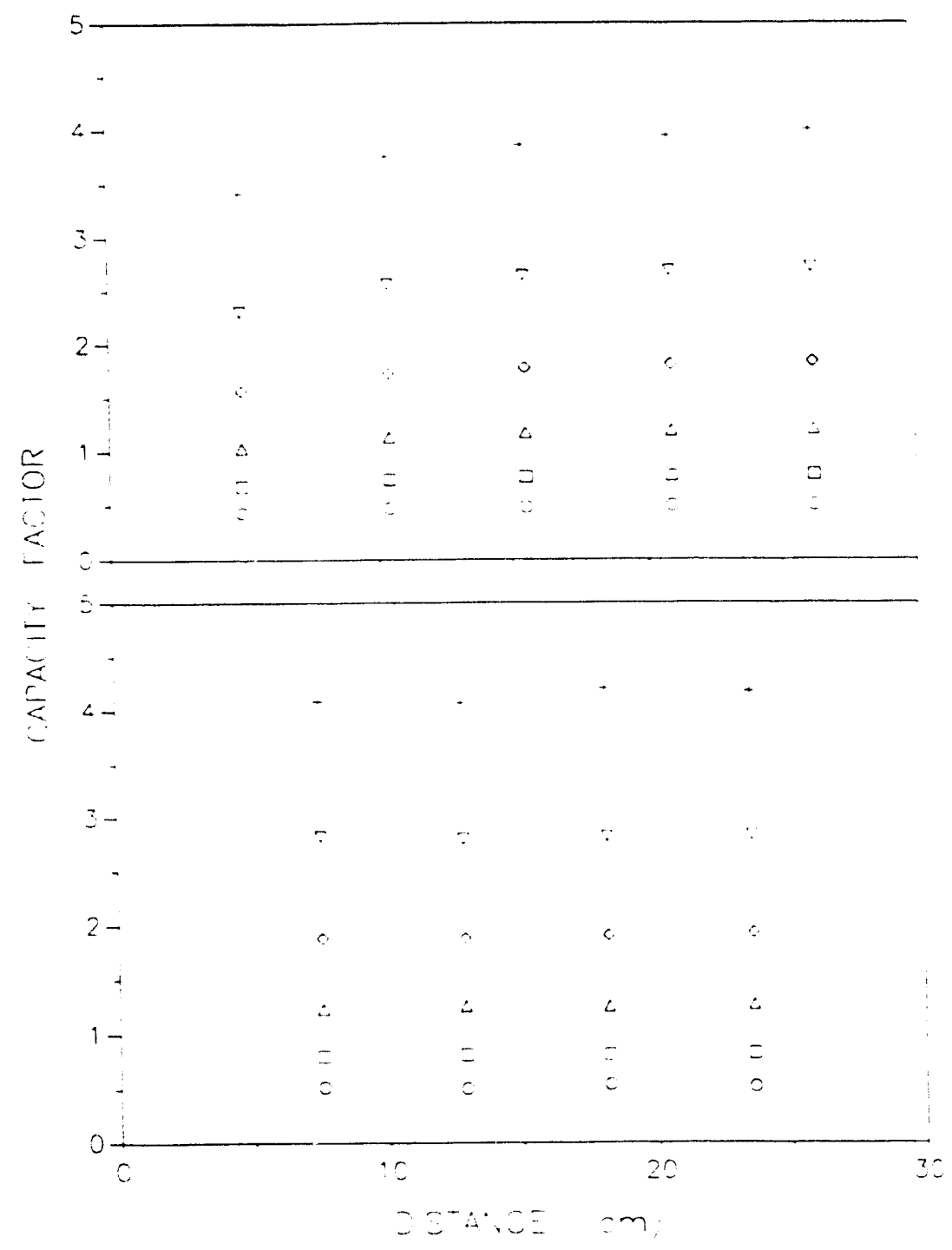

Figure 2

Single- (top) and dual- (bottom) mode measurements of solute capacity factor as a function of distance along the column ior an inlet pressure of 102 bar. Solutes: $n-C_{10: 0}(O) ; n-C_{12: 0}(\square) ; n-C_{14: 0}(\Delta) ; n-C_{16: 0}(O) ; n-C_{18: 0}(\nabla) ; n-C_{20: 0}(+)$. 
To isolate the influence of local pressure on solute retention, it is necessary to maintain all other parameters constant while varying the local pressure on the column. As described earlier, this is experimentally accomplished using a restrictor at the column exit to alter the local pressure, while the splitter length is varied to maintain the pressure gradient and the volumetric flowrate at constant levels. As illustrated in Figure 3, both single- (top) and dual- (bottom) mode measurements indicate that solutes are well behaved under these conditions. Even at the highest inlet pressure, solutes exhibit the logarithmic dependence of solute capacity factor on carbon number expected based solely on dispersion interactions. Moreover, each solute shows an apparent increase in capacity factor with inlet pressure.

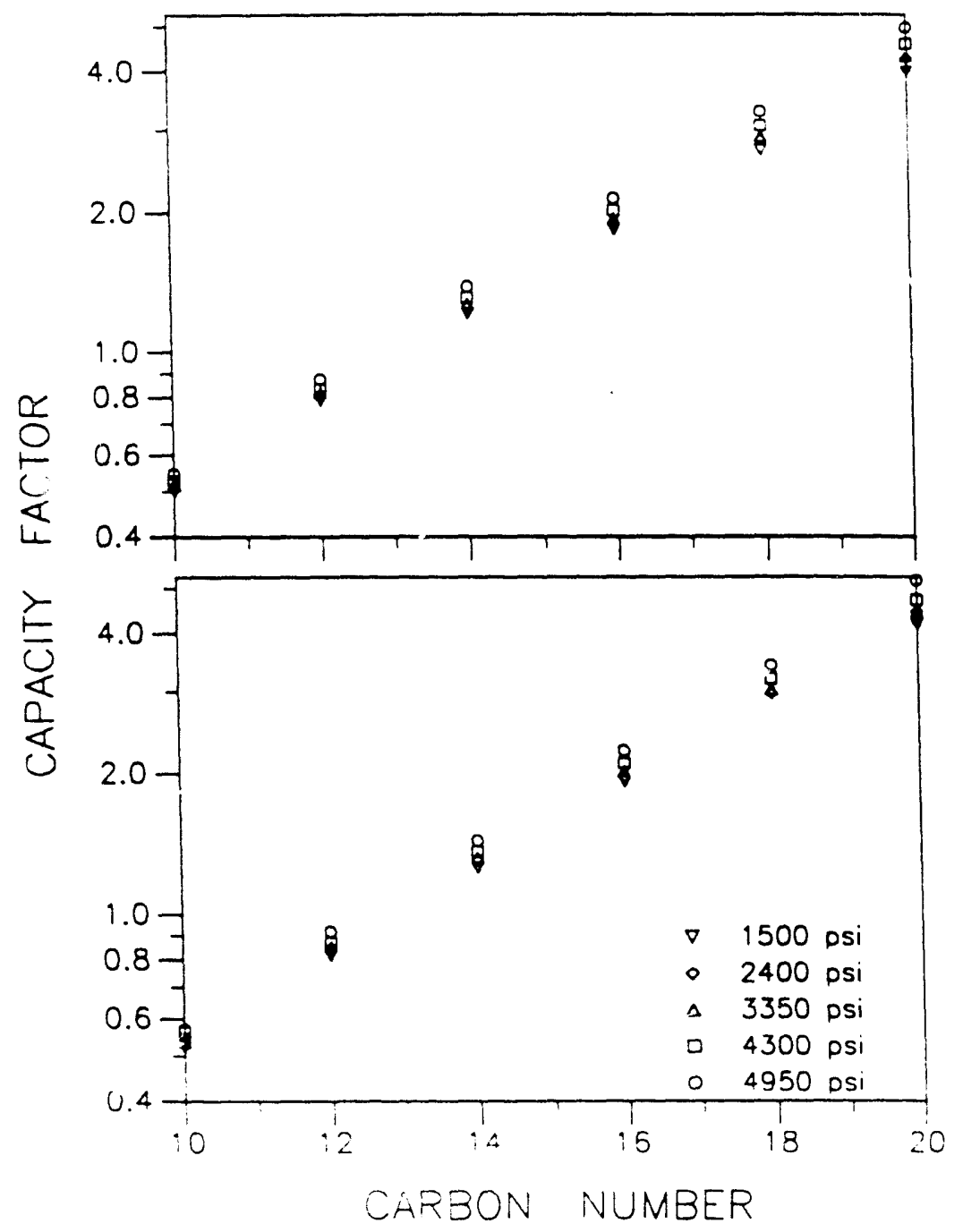

Figure 3

Single- (top) and dual- (bottom) mode measurements of logarithmic dependence of capacity factor on carbon number under varying inlet pressure conditions. 
This increase in retention with the average pressure encountered by the solute is clearly illustrated in Figure 4 for both single- and dual-mode measurements $(L=26.2$ and 23.5 $\mathrm{cm}$, respectively). A significant, nonlinear increase in the retention of $n-C_{16: 0}$ is observed as a function of pressure for both single and dual modes, with $n-C_{16: 0}$ exhibiting an increase of greater than $16 \%$ with a precision in duplicate measurements of $0.5 \%$. This increase is not limited to $n-C_{16: 0}$, and a notable increase in $k$ is measured for all solutes under practical inlet conditions (Table 1). Although all solutes exhibit this increase in retention with pressure, the experimentally measured percent increase $(\Delta \mathrm{k} / \mathrm{k})$ is systematically greater for longer chain solutes. Thus, the influence of pressure on solute retention appears to be a complex function of both solute and mobile-phase properties.

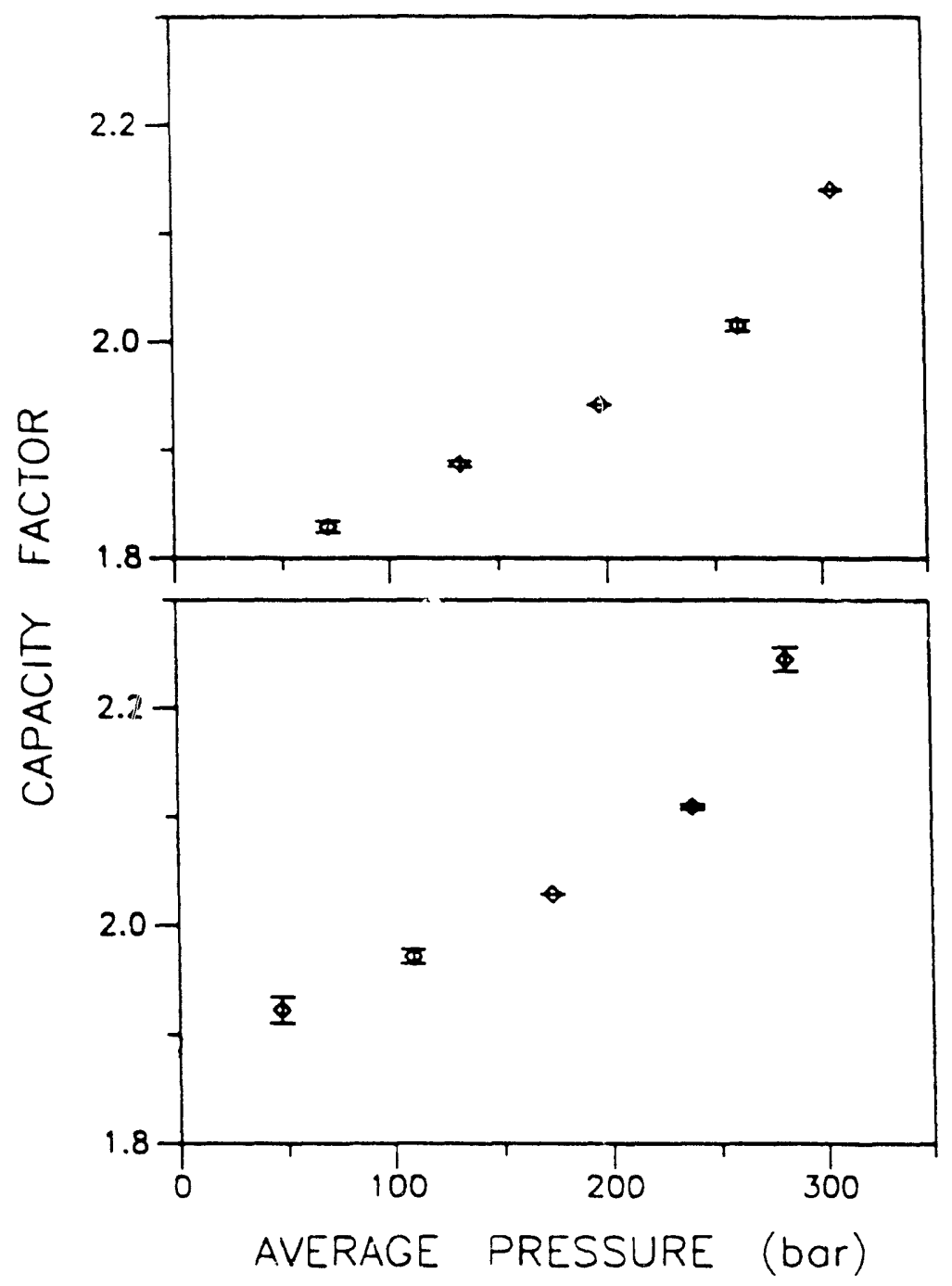

Figure 4

Single- (top) and dual- (bottom) mode measurements of solute capacity factor ( $L=26.2$ and $23.5 \mathrm{~cm}$, respectively) for $n-\mathrm{C}_{16: 0}$ as a function of the average pressure encountered by the solute. 
Table I

Effect of pressure on single- and dual-mode measurements of solute capacity factor.

\begin{tabular}{|c|c|c|c|c|c|c|}
\hline \multirow[b]{3}{*}{ SOLUTE } & \multicolumn{5}{|c|}{ CAPACITY FACTOR (k) } & \\
\hline & \multicolumn{3}{|c|}{ SING'_E MODEa } & \multicolumn{3}{|c|}{ DUAL MODEb } \\
\hline & $P_{A V G}=72$ bar & 236 bar & $\Delta k / k$ & $48 \mathrm{bar}$ & $158 \mathrm{bar}$ & $\Delta k / k$ \\
\hline$n-C_{10: 0}$ & 0.501 & 0.547 & $+9.2 \%$ & 0.520 & 0.571 & $+9.8 \%$ \\
\hline$n-C_{12: 0}$ & 0.784 & 0.874 & $+11.5 \%$ & 0.818 & 0.921 & $+12.6 \%$ \\
\hline$n-C_{14: 0}$ & 1.208 & 1.383 & $+14.5 \%$ & 1.261 & 1.441 & $+14.3 \%$ \\
\hline$n-C_{16: 0}$ & 1.829 & 2.141 & $+17.1 \%$ & 1.923 & 2.245 & $+16.8 \%$ \\
\hline$n-C_{18: 0}$ & 2.712 & 3.284 & $+21.1 \%$ & 2.827 & 3.419 & $+20.9 \%$ \\
\hline$n-C_{20: i}$ & 3.997 & 4.970 & $+24.3 \%$ & 4.197 & 5.205 & $+24 .\{\%$ \\
\hline
\end{tabular}

a $L=26.2 \mathrm{~cm}$

b $L=23.5 \mathrm{~cm}$

A qualitative understanding of this phenomenon can be provided by the solubility parameter model $(18,19)$, a thermodynamic approach based on regular solution theory. The solubility parameter $(\delta)$ of a substance is defined as the cohesive energy density or interaction energy $(E)$ per molar volume (' $V$ ):

$\delta^{2}=-E N$

This quantity, which is a direct measure of polarity, can be related to the capacity factor of solute $i$ by the following equation (20):

$\ln k=\left(V_{i} / R T\right)\left[\left(\delta_{i}-\delta_{m}\right)^{2}-\left(\delta_{i}-\delta_{s}\right)^{2}\right]-\ln \beta$

Solute retention can thus be expressed in terms of the solubility parameters of the solute $\left(\delta_{i}\right)$, the mobile phase $\left(\delta_{m}\right)$, and the stationary phase $\left(\delta_{s}\right)$, as well as the volume ratio of the mobile and stationary phases $(\beta)$. For reversed-phase separations, the mobile phase is always more polar than the stationary phase, and the solute is generally of intermediate poiarity, so that $\delta_{\mathrm{m}}>\delta_{\mathrm{i}}>\delta_{\mathrm{s}}$. As the polarity of the solute approaches that of the mobile phase, $\delta_{1}-\delta_{m}$ decreases and In k decreases; conversely, as the polarity of the solute approaches that of the stationary phase, $\ln \mathrm{k}$ increases. An increase in pressure is often assumed to decrease the molar volume, without altering the nature or energy of 
interaction (19). While this assumption may be questionable for molecular species with multiple types of interactions, it appears to be reasonable if only dispersion interactions are present. Thus, pressure is predicted to yield an increase in the cohesive energy density and solubility parameter, according to Equation [2]. If pressure variations are considered to influence both the mobile and stationary phases, $\delta_{m}$ and $\delta_{\varepsilon}$ are expected to increase with pressure. Thus, solute interautions with the stationary phase are expected to increase with pressure, whereas interactions with the mobile phase are predicted to decrease. Based on Equation [3], solute retention should systematically increase with pressure for reversed-phase separations. By using similar arguments, solute retention is expected to decrease with pressure for normal-phase separations $\left(\delta_{s}>\delta_{i}>\delta_{m}\right)$, as observed by Katz et al. (11). Although these conclusions are in qualizative agreement with experimental measurements, this phenomenon is clearly quite complex and warrants more detailed theoretical consideration.

\section{CONCLUSIONS}

Direct examination of solute retention under varying pressure conditions indicates a compirax dependence of the solute capacity factor on the local pressure present on the chromatographic column. Under pressure conditions typical for liquid chromatographic separations, the capacity factor of model solutes increases significantly with the average pressure encountered by the solute. The magnitude of this increase is somewhat surprising based on the common belief that reversed-phase solvents are quite incompressible. It is even more surprising for these model solutes, where only dispersion interactions are controlling retention. Bevause these universal interactions are present in all separations, the variation in retention with pressure has clear implications for the practice of liquid chromatography as well as the measuremert of fundamental chromatographic parameters.

\section{ACKNOV LEDGEMENTS}

This research was supported by the U.S. Department of Energy, Office of Basic Energy Sciences, Division of Chemical Sciences under contract number DE-FG02-89ER14056. 


\section{LITERATURE CITED}

1. J.C. Giddings; in Gas Chromatography 1964; A. Goldup Ed.; Elsevier: Amsterdam, 1965.

2. J.C. Giddings; Separ. Sci. 1 (1966) 73.

3. J.C. Giddings, M.N. Myers, L. McLaren and R.A. Keller; Science 162 (1968) 67.

4. R.C. Reid, J.M. Prausnitz and T.K. Sherwood; The Properties of Gases and Liquids; McGraw-Hill: New York, 1977.

5. K. Stephans and K. Lucas; Viscosity of Dense Fluids; Plenum: New York, 1979.

6. P.E. Liley, T. Makita and Y. Tanaka; Properties of Inorganic and Organic Fluids; Vol. V-1; Hemisphere Publishing: New York, 1988.

7. D.E. Martire; J. Liq. Chromatogr. 10 (1987) 1569.

8. B.A. Bidlingmeyer, R.P. Hooker, C.H. Lochmuller and L.B. Rogers; Separ. Sci. 7 (1972) 131.

9. $\quad$ G. Prukop and L.B. Rogers; Separ. Sci. 13 (1978) 59.

10. N. Tanaka, T. Yoshimura and M. Araki; J. Chromatogr. 406 (1987) 247.

11. E. Katz, K. Ogan and R.P.W. Scott; J. Chromatogr. 260 (1983) 277.

12. C.E. Evans and V.L. McGuffin; Anal. Chem. 60 (1988) 573.

13. C.E. Evans and V.L. McGuffin; J. Liq. Chromatogr. 11 (1988) 1907.

14. C.E. Evans and V.L. McGuffin; J. High Res. Chromatogr. Commun.; submitted.

15. V.L. McGuffin and R.N. Zare; Appl. Spectrosc. 39 (1985) 847.

16. J.C. Gluckman, A. Hirose, V.L. McGuffin and M. Novotny; Chromatographia 17 (1983) 303.

17. B.A. Bidlingmeyer and F.V. Warren; Anal. Chem. 56 (1984) 1583A.

18. J.H. Hildebrand and R.L. Scott; Solubility of Nonelectrolytes; Reinhold: New York, 1950.

19. A.F.M. Barton; Chem. Rev. 75 (1975) 731.

20. P.J. Schoenmakers, H.A.H. Billiet and L. de Galan; Chromatographia 15 (1982) 205. 

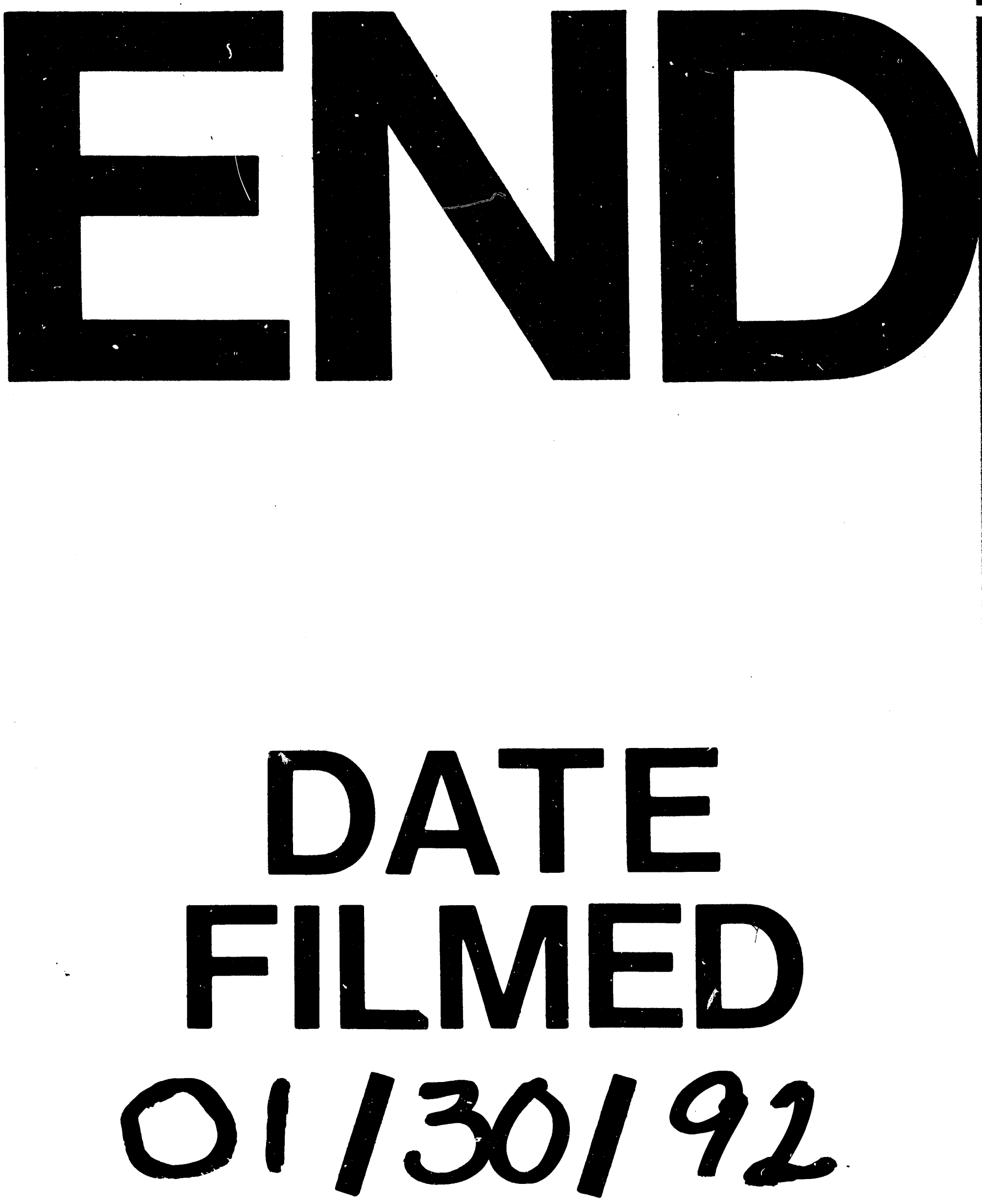